\title{
What is scientific understanding and how can it be achieved?
}

\author{
Henk W. de Regt and Christoph Baumberger
}

Science has not only produced a vast amount of knowledge about a wide range of phenomena, it has also enhanced our understanding of these phenomena. Indeed, understanding can be regarded as one of the central aims of science. But what exactly is it to understand phenomena scientifically, and how can scientific understanding be achieved? What is the difference between scientific knowledge and scientific understanding? These questions are hotly debated in contemporary epistemology and philosophy of science. While philosophers have long regarded understanding as a merely subjective and psychological notion that is irrelevant from an epistemological perspective, nowadays many of them acknowledge that a philosophical account of science and its aims should include an analysis of the nature of understanding. This chapter reviews the current debate on scientific understanding. It presents the main philosophical accounts of scientific understanding and discusses topical issues such as the relation between understanding, truth and knowledge, the phenomenology of understanding, and the role of understanding in scientific progress.

\section{Introduction}

Science has not only produced a vast amount of knowledge about a wide range of phenomena, from the nature of elementary particles to the structure of the universe, but it has also enhanced our understanding of these phenomena. Indeed, understanding can be regarded as one of the central aims of science. Moreover, scientific understanding is not only important for its own sake, but also highly relevant to society. Climate scientists, for example, want to understand the process of global warming and other climate changes, because such understanding is a first, necessary step towards solving today's environmental problems. Accordingly, the main task of the Intergovernmental Panel on Climate Change (IPCC) is to assess progress in scientific understanding of climate change, as explicitly stated in their latest report Climate Change 2013: The Physical Science Basis (IPCC 2013, p.4).

But what exactly is scientific understanding, and how can it be achieved? These questions are hotly debated in contemporary epistemology and philosophy of science. While philosophers have long regarded understanding as merely subjective and psychological notion that is irrelevant from an epistemological perspective, nowadays many of them acknowledge that a philosophical account of science and its aims should include an analysis of the nature of understanding. This chapter reviews the current debate on scientific understanding. We first present the main philosophical accounts of scientific understanding, and we then discuss topical issues such as the relation between understanding and knowledge, the phenomenology of understanding, and the role of understanding in scientific progress. 


\section{The contextual theory of scientific understanding}

In the current debate on the nature of scientific understanding, there appear to be two types of approaches. On the one hand, some philosophers emphasize that scientific understanding should ultimately be grounded in objective scientific explanations or knowledge of, for example, causal relations, where understanding consists in a 'grasp' of those explanations or causal relations (Grimm 2017; Khalifa 2017; Strevens 2013). Although they acknowledge that understanding involves a 'grasp', which is a cognitive achievement that relates to the psychology of the subject, they do not see grasping as a sufficient condition for genuine scientific understanding. On the other hand, there are philosophers who put the pragmatics of understanding center stage in their analysis. This typically leads to approaches that invoke the results of empirical study by, for example, psychologists, historians, or sociologists of science. Thus, Faye (2014) bases his pragmatic-rhetorical theory of (scientific) understanding in part on results from cognitive science and evolutionary theory, while De Regt (2017) has developed his contextual theory of scientific understanding on the basis of historical case studies of scientific development. In this section we will outline De Regt's theory, which was one of the first fullfledged theories of scientific understanding that appeared on the scene, in more detail. In the next section, we will come back to the more objectivist approaches mentioned erlier.

De Regt's theory is based on the analysis of examples from the history of science (esp. physics) and on recent insights that philosophers of science have derived from studying scientific practice. Its central idea is the thesis that scientists achieve understanding of a phenomenon $P$ if they construct an appropriate model of $P$ on the basis of a theory $T$, following the modelbased account of explanation defended by Cartwright (1983, pp.143-162). More specifically, the contextual theory is built upon a Criterion for Understanding Phenomena (De Regt 2017, p.92):

CUP: A phenomenon $P$ is understood scientifically if and only if there is an explanation of $P$ that is based on an intelligible theory $T$ and conforms to the basic epistemic values of empirical adequacy and internal consistency.

The key term in this criterion is 'intelligible': understanding of phenomena requires an intelligible theory, where intelligibility is defined as (De Regt 2017, p.40):

Intelligibility: the value that scientists attribute to the cluster of qualities of a theory $T$ (in one or more of its representations) that facilitate the use of $T$.

This definition entails that intelligibility is not an intrinsic property of theories, but a contextdependent value: whether or not a theory is intelligible to scientists depends on, for example, their skills and their background knowledge. Why do scientific theories need to be intelligible to the scientists who use them? De Regt's argument for this claim draws on the work of philosophers Nancy Cartwright (1983) and Margaret Morrison (1999, see esp. pp.60-64), who highlighted the pivotal role of modelling in scientific practice, and in explanatory practices in particular. On the model-based account of scientific explanation scientists acquire understanding of the phenomena by constructing models, which 'mediate' between relevant theories 
and the phenomenon-to-be-explained. Constructing such mediating models involves pragmatic judgments and decisions, since models do not follow straightforwardly from theories (and neither do they follow from the empirical data). For example, suitable idealizations and approximations need to be made. De Regt submits that the construction of such models - which provide explanatory understanding of phenomena - requires theories that are intelligible in the sense defined above. Only if scientists' ability to work with the theory allows them to make suitable pragmatic judgments, will they succeed in constructing explanatory models. In sum, understanding a phenomenon on the basis of $T$ depends on an appropriate combination of skills of $S$ and qualities of $T$.

An example of such a quality is visualizability. This is a theoretical quality that is widely valued, because for many scientists visualizable theories are more tractable and easier to work with (De Regt 2014). But the contextual theory does not imply that visualizability is a necessary condition for the intelligibility of scientific theories. Depending on the context, there may be alternative ways to render theories intelligible. ${ }^{1}$ A concrete example of model construction in which visualization plays a role is the explanation of gas phenomena on the basis of the kinetic theory of gases, as it was developed by James Clerk Maxwell and Ludwig Boltzmann in the nineteenth century. The kinetic theory represents gases as aggregates of particles (molecules) in motion obeying the laws of Newtonian mechanics. Specific models of the molecules and their structure have to be constructed in order to explain particular gas phenomena on the basis of the theory. For example, the kinetic explanation of Boyle's law involves the construction of a model (the ideal gas model) that represents gas molecules as point masses, such that application of Newton's laws leads to a theoretical prediction of the relationship between pressure and volume. The ideal gas model does not follow deductively from the kinetic theory, as its construction involves idealizations and approximations. Specific features of the theory - its visualizability, but also its causal aspects - guaranteed its intelligibility to Maxwell and Boltzmann (and to physicists ever since). ${ }^{2}$ Subsequently, the construction of more specific molecular models, such as the van der Waals model and the dumbbell model for diatomic gases, has yielded additional or more detailed understanding of gas phenomena. ${ }^{3}$

\section{Alternative accounts of scientific understanding}

According to the contextual theory introduced in the previous section, scientific understanding of a phenomenon requires the ability to use a theory to construct (a model through which one can derive) an explanation of the phenomenon that conforms to the epistemic values of empirical adequacy and logical consistency. Alternative accounts of understanding are typically more demanding in what they require from the explanation and less demanding with respect to the abilities they require from the scientist. ${ }^{4}$ Many of them identify understanding with 'grasping' a correct and thus an at least approximately true explanation. Grasping an explanation is distinguished from merely believing or even from knowing the explanation, but it does not require being capable of using a theory to construct the explanation from scratch. The accounts differ in how they conceive of the grasping that they take to be characteristic of scientific understanding. 
Stephen Grimm (2006; 2010) suggests that the distinction between grasping and believing an explanation lies in one's ability to answer counterfactual 'what-if-things-had-been-different' questions. This, in turn, is the ability to anticipate the sort of changes that would result if the factors cited as explanatory were different in various ways. Understanding why a certain plane can fly, for example, requires not only the ability to see how Bernoulli's principle applies to the relevant details about the plane and thus to recognize that the shape of its wings (curved on the top and flat on the bottom) creates a difference in the velocity of air and thus in the pressure exerted along the top and the bottom of the wings. One needs also be able to anticipate how changes, for instance in the velocity, would lead to a change in the pressure of the air, and, as result, see that if the top of the wings were flattened out, the plane would not be able to fly anymore (Grimm 2010, pp.340-341). Since one can be able to reason counterfactually about an explanation in this way without being capable of actually deriving the explanandum, Grimm's condition of grasping is less demanding than De Regt's requirement of intelligibility.

Mark Newman $(2012 ; 2017)$ agrees that grasping a scientific explanation involves abilities that are not required for believing and for knowing the explanation. He deems central the ability to draw correct inferences about why the explanans explains the explanandum; for example, why the staying aloft of a plane is a consequence of Bernoulli's principle being applied to the relevant details about the plane. Newman (2012) has developed this idea in his Inferential Account of Scientific Understanding. However, he argues that the grasping necessary for scientific understanding requires neither the ability to construct an explanation, nor the ability to apply it to counterfactual cases and solve new problems. Newman (2017) suggests that if someone has such problem-solving abilities as well, we should rather say that she understands a theory, which she can use to comprehend a whole range of phenomena.

Strevens $(2013 ; 2017)$ also identifies explanatory understanding with grasping a correct scientific explanation, but ascribes abilities a less prominent role than Grimm and Newman. Strevens takes grasping to be "the fundamental relation between mind and world" (2013, p.511). He does not give an account of this relation but suggests that it involves a more intimate epistemic acquaintance than knowledge. By way of testimony, you might know Bernoulli's principle, the relevant details about a plane, and that the possibility of flight can be deduced from them, even if you comprehend only dimly the content of your beliefs. Grasping the explanation requires a firmer grip on the explanatory connections that enables you to see why the principle together with the details about the plane explain why the plane can fly. This idea is familiar from Newman. But whereas Newman identifies the grasping required for scientific understanding with having inferential abilities, Strevens (2017, p.41) suspects that this gets the order of dependence wrong and suggests that the abilities are grounded in the psychological state of grasping.

Unlike the previous accounts, Kareem Khalifa (2017) explicates explanatory understanding with reference to propositional knowledge and acknowledges that understanding admits of degrees. He combines an account of minimal understanding with two comparative principles of understanding. Minimal understanding of why a phenomenon occurs is identified with believing an approximately true explanation of the phenomenon. The first comparative principle says that one person understands better why a phenomenon occurs than another if she 
has a more complete grasp of the correct explanations of the phenomenon and the relations between them. How complete her grasp is, depends on the number, the quality, and the level of detail of the explanations. The second comparative principle says that one person understands better why a phenomenon occurs than another if her grasp of the phenomenon's explanations and their interrelations bears greater resemblance to scientific knowledge than the second person's. Scientific knowledge of why a phenomenon occurs is based on a scientific evaluation of the explanations. Such an evaluation considers many plausible potential explanations for the phenomenon, compares those using scientific methods, and assigns an appropriate degree of belief for the explanations based on the comparison. Grasping explanations is on this view simply a cognitive state that resembles scientific knowledge of explanations. How close the resemblance is, depends on the number of plausible potential explanations that one has considered and compared, the scientific status of the methods used in this comparison, and the safety and accuracy of the resulting beliefs.

The accounts introduced so far are accounts of explanatory understanding: the understanding of why a phenomenon occurs that results from a scientific explanation of that phenomenon. Explanatory understanding can be distinguished from the more holistic objectual understanding. Here, scientists seek more than a single explanation of a phenomenon that is defined in terms of a small set of salient features and use a theory or model to understand a bunch of phenomena or a system. Examples are understanding climate change through climate models, or the origin of species in terms of evolutionary theory. In his Understanding as Representation Manipulability account, Daniel Wilkenfeld (2013) suggests that to understand a system is to have a mental representation of it that one can modify in such a way that enables one to manipulate or make relevant inferences about the system. The ability to modify a representation consists in being able to correct minor errors in one's representation and apply it to similar cases to cast predictions and give explanations. Wilkenfeld (2017) conceives of the ability to make relevant inferences about a system as an evaluative criterion rather than a necessary condition for scientific understanding, and suggests representational accuracy as a further criterion for assessing how good someone's understanding is. Building on this proposal, Baumberger (forthcoming) adds justification as a third evaluative criterion and commitment as a necessary condition for objectual understanding (cf. Elgin 2017, p.44). Taking the ability to make relevant inferences, representational accuracy and justification as evaluative criteria rather than necessary conditions accommodates the insight that these dimensions play an important role in the ascription and assessment of scientific understanding, without denying that there may be contexts in which we rightly ascribe understanding while some of the conditions are hardly met or not met at all.

\section{Key issues in the current debate about scientific understanding}

The accounts that we discussed above raise a number of systematic issues. For example, is understanding a form of knowledge, as some accounts assume? How are understanding and the grasping that many accounts take to be essential for understanding related to the 'feeling' or 
'sense' of understanding? What is the role of understanding in scientific progress, and how should we account for this role? These questions are addressed in the following paragraphs.

\section{Understanding, truth and knowledge}

You can obviously know that a phenomenon occurs without understanding why it occurs, but is it also possible to have knowledge-why without understanding-why? Not for knowledgebased accounts that identify understanding with explanatory knowledge. An example is Peter Lipton (2004, p.30) who declared: "Understanding is not some sort of super knowledge, but simply more knowledge: knowledge of causes." Philosophers who take explanatory understanding to be more demanding than explanatory knowledge typically claim that the former involves abilities that are not necessary for the latter, for example, the ability to use a theory to construct an explanation (De Regt), the ability to draw inferences about why the explanans explains the explanandum (Newman), or the ability to engage in counterfactual reasoning (Grimm).

Defenders of knowledge-based accounts object that some of these abilities are not necessary for (minimal) understanding, and those that are necessary are already required for explanatory knowledge (Khalifa 2017, pp.54-60). It seems, for example, possible to have some understanding of a phenomenon even if one is neither able to construct an explanation, nor to draw inferences about why the explanans explains the phenomenon. The ability to engage in counterfactual reasoning, on the other hand, seems already necessary for explanatory knowledge. Even believing an explanation requires that one is able to answer some what-if questions (Grimm 2014, p.388). For example, believing that the lift of a plane depends (according to Bernoulli's principle) on a difference in the velocity of air traveling over the wings, requires being able to see that had there been no difference in velocity, there would not have been a difference in pressure and hence no lift. Thus, (minimal) understanding does not seem to involve abilities that are categorically distinct from those required for believing an explanation. Good or deep understanding may involve additional abilities, but they might also be required for more demanding instances of scientific knowledge-why (Khalifa 2017, pp.61-63).

Whether some form of knowledge is sufficient for understanding is thus mainly a question of whether the second requires abilities that are not required for the first. A further issue is whether knowledge is necessary for understanding. Is it for instance possible to understand why a phenomenon occurs without knowing why it occurs? This depends on our notion of knowledge. Epistemologists typically conceive of knowledge as justified true belief, the truth of which is not due to epistemic luck. For each necessary condition for knowledge, it has been argued that it is not necessary for understanding. Some have argued that understanding is compatible with certain types of epistemic luck that undermine knowledge. ${ }^{5}$ Others have suggested that, in contrast to knowledge, understanding requires neither belief nor justification. ${ }^{6}$ However, the liveliest debate about a possible divergence between knowledge and understanding concerns the question of whether understanding implies truth.

Knowledge is factive: if one knows that $p$, then $p$ is true. If understanding is a form of knowledge, understanding must be factive too. Since scientific explanations are often complex 
and involve for instance initial conditions and generalizations, a factivity condition for explanatory understanding requires that all propositions constituting the explanation be true. Adapted to objectual understanding such a condition requires that all propositions constituting one's representation of the target system be true. At least for objectual understanding, such a strong factivity condition seems too demanding. A few peripheral falsehoods may degrade one's understanding, but do not undermine it completely. Thus, moderate factivists only require that all central propositions be true (Kvanvig 2003, pp.201-202).

Non-factivists argue that scientific understanding is not even moderately factive. They point out that we gain understanding through idealized models (e.g., the ideal gas model) and superseded theories (e.g., Newton's theory of gravitation), even though both contain nonperipheral falsehoods (e.g., the assumption that a gas consists of perfectly elastic point masses that do not interact with each other in the case of the ideal gas model, and the assumption that there are gravitational forces in the case of Newton's theory of gravitation). Moreover, we can understand phenomena in terms of non-propositional representations such as diagrams and material models, which are not even truth-apt (De Regt 2015; Elgin 2017).

Moderate factivists can pursue two strategies to save factivity (Baumberger, Beisbart and Brun 2017, pp.8-10). First, they can argue that the alleged counterexamples do not display genuine understanding. The idea is that if we ascribe understanding in such cases, we use the term honorifically, as when we speak of "the current state of scientific knowledge" while conceding that part of it may be false (Greco 2014, pp.297-298). However, since at least idealized models do hardly preclude genuine understanding, the second strategy seems more promising. It claims that in those examples that display genuine understanding, moderate factivity is not really violated. Understanding a phenomenon with an idealized model, for example, requires that a scientist knows what idealizations the model involves, which aspects of the phenomenon it is intended to describe, and under which conditions the phenomenon approximately behaves as the model. If a scientist knows all this, her central beliefs about the phenomenon are true (Greco 2014, pp.296-297). In the ideal gas case, we need to distinguish between the ideal gas law, the conditions for its application, and the idealizing assumptions that are needed to derive the ideal gas law. These assumptions (e.g., that the particles do not interact) are indeed false, but they are at the periphery of the model since they do not belong to the description of the behavior of real gases. This behavior is rather characterized by the ideal gas law. The law and its conditions of applicability constitute the central propositions of the model. Since in successful applications of the model, the conditions are satisfied and the law is approximately true, moderate factivity seems to hold (Mizrahi 2012; cf. Khalifa 2017, pp.173-175).

Non-factivists can respond to this defense by pointing out that in some cases, we credit scientists with an understanding of a phenomenon even though they do not exactly know how their models diverge from the phenomenon or under which conditions the models provide an approximately true description of the phenomenon. Moreover, De Regt (2015) suggests examples from economy and ecology, in which scientists acquire understanding by applying models whose central proposition are not even approximately true.

Factivists and non-factivists face different challenges. Factivists need to explain how idealized models and flawed theories can contribute to understanding. To meet this challenge, 
Strevens (2017) argues that idealizations enhance understanding by highlighting that certain factors make no difference to the explanandum, and that we can learn why other factors are difference-makers by manipulating idealized models. Non-factivists, on the other hand, need to explain why we cannot gain understanding by any kind of just-so story or false theory. To meet this challenge, De Regt and Gijsbers (2017) suggest that representations provide understanding only if they reliably lead to scientific success, i.e. to true predictions, successful practical applications, and fruitful ideas for further research. Newton's theory of gravitation enables us to understand certain phenomena because it is very successful in a broad range of applications. Understanding and truth are thus connected for De Regt and Gijsbers, but what needs to be true are predictions rather than the theories or models themselves.

\section{The phenomenology of understanding}

Understanding as insight, or the grasping of an explanation, is often associated with a so-called Aha-feeling, a Eureka-moment of the kind experienced by Archimedes when he took a bath and suddenly understood how he could find out whether or not the crown of the king was made of pure gold, thereby discovering the law of buoyancy that was later named after him. The emotion he experienced was so strong that he allegedly jumped out of his bath and ran naked through the streets of Syracuse, shouting "Eureka!": "I have found it!" The story shows that understanding can come with a particular phenomenology. While interesting from a psychological point of view, one may wonder what the philosophical import of the phenomenology of understanding is. Does the feeling or 'sense' of understanding carry any epistemic weight, and should it therefore be included in a philosophical theory of understanding? Opinions diverge among contemporary philosophers of science and epistemologists.

The debate started with a provocative paper by J.D. Trout (2002), who argued that the sense of understanding is a highly unreliable feeling that is prone to cognitive biases such as the hindsight bias and the overconfidence bias. ${ }^{7}$ Accordingly, scientists should never trust feelings of understanding such as Archimedes' Eureka-experience. And philosophers wanting to develop a theory of scientific explanation should stay away from the notion of understanding. The reason is, Trout stated, that the phenomenology of understanding does not give a clue as to whether the explanation that gives rise to it is actually correct. Trout suggested that the history of science is replete with examples of scientists experiencing a feeling of understanding and yet being completely wrong, and he cited Ptolemy as a case in point.

Trout's claim is confirmed by empirical studies in cognitive psychology, carried out by Leonid Rozenblit and Frank Keil, which reveal the existence of a so-called illusion of explanatory depth: "People feel they understand complex phenomena with far greater precision, coherence, and depth than they really do" (Rozenblit and Keil 2002, p.521; cf. Keil 2006). It can be argued that there is no reason to assume that scientists are less prone to this illusion than people in general, and that scientific understanding may therefore be equally biased (Ylikoski 2009).

Most philosophers agree that these empirical results show that the feeling or sense of understanding should be distinguished from the understanding itself, or "understanding proper", as Kuorikoski (2012) has called it. Still, the results do not entail an unambiguous 
conclusion about the relation between the two, since that obviously also depends on which conception of "proper understanding" is adopted. ${ }^{8}$ If one assumes that proper understanding can be reduced to explanatory knowledge (as, e.g, Trout and Khalifa have suggested), then the feeling of understanding appears to be neither necessary, nor sufficient for proper understanding and thereby irrelevant for philosophical theories of understanding. But if proper understanding involves some kind of grasping, it is not a priori clear how it relates to the feeling of understanding since grasping is a cognitive, psychological state that may come with a particular phenomenology. As we have seen, many authors associate grasping with some kind of ability, for example to construct models of the objects of understanding (De Regt), to make counterfactual inferences about them (Newman, Kuorikoski), or to manipulate representations of them (Wilkenfeld 2013). As Grimm (2009, p.85) notes, "at a more basic level the 'sense of understanding' seems to refer to the exercise of an ability or faculty that undergirds the phenomenology", and it is an open question whether our sense of understanding is reliable in the sense that the distinctive phenomenology of understanding typically leads us to a correct grasp of the objects of understanding.

Grimm (2009) answers this question in the affirmative, arguing that the feeling of understanding can serve an epistemic function. He admits that it is not reliable per se, but argued that it is 'conditionally reliable', that is, it is "reliable so long as our background beliefs are more or less sound, our intellectual practices are more or less virtuous, and so on" (Grimm 2009, p.93). Scientists are most likely to feel that they understand something when that bit of information coheres with the rest of their beliefs about the world. While this does not guarantee the reliability of the feeling of understanding, it does imply that it can be checked by reviewing one's background knowledge.

Also Lipton (2009, pp.54-60) argued that although the subjective feeling of understanding should be sharply distinguished from objective proper understanding, the former is not irrelevant to the latter. To begin with, feelings such as the Eureka-experience work as an incentive: the prospect of such pleasant feelings can motivate the search for proper understanding (cf. Gopnik 2000). Moreover, the feeling of understanding may guide our practice of inference to the best explanation, as Lipton held that the best explanation is typically the "loveliest" explanation: the explanation which, if correct, would provide the most understanding. ${ }^{9}$ Of course, this does not by itself prove that the feeling of understanding is a reliable guide, but Lipton (2009, pp.59-60) suggested that just as our perceptual system is mediated by subjective experience and yet is a reliable source of knowledge, our subjective experiences of understanding may be "well calibrated and a reliable guide to theory choice".

In conclusion, while there is general agreement that proper (scientific) understanding should be distinguished from its phenomenology, there is an ongoing debate over the question of whether the feeling of understanding is at least to some extent reliable as a cue to proper understanding and has accordingly epistemic value. This is both an empirical and a conceptual matter and merits further investigation on both fronts. 


\section{Understanding, the history of science, and scientific progress}

Since it is generally accepted that science, in the course of its historical development, has progressed enormously, and since progress in science can be defined as increasing success in achieving its aims, it seems obvious that scientific progress involves increase in understanding. Surprisingly, however, it is hard to find statements to this effect in the work of philosophers of science. The reason may be that although few philosophers will deny that science has progressed, there is no agreement about the nature of scientific progress, and philosophers of science have debated the question of what it exactly consists in for decades. In this section, we will focus on the relation between scientific progress and scientific understanding. Does scientific progress involve, or even consist in, an increase of understanding? Or does progress comprise something else, for example, increase of knowledge or of problem-solving power, or an ever closer approximation to the truth? In the latter case, it is not a priori true that progress in science implies an improvement of (increase in) understanding.

As mentioned, it is remarkable that the relation between progress and understanding has not received much explicit attention in the philosophical literature until very recently. Most earlier accounts of scientific progress were framed in terms of the concepts listed above: knowledge, problem-solving power, or truth-approximation. ${ }^{10}$ A recent exception is Angela Potochnik, who in her 2017 book Idealization and the Aims of Science defends the claim that understanding rather than truth is the key aim of science and suggests that this allows for a more convincing account of progress in science. She argues that accounts of scientific progress in terms of truth-approximation face at least two problems that suitable understanding-based accounts do not (Potochnik 2017, p.121). The first is the so-called pessimistic meta-induction, which implies that our current scientific theories may be radically false, such that no convergence to the truth has been achieved so far. The second is the crucial role of idealizations in science, which suggests that progress can be made by means of departing from the truth. Potochnik claims that an account of scientific progress in terms of increase in understanding would avoid these problems.

Whether that is the case depends of course on the conception of scientific understanding that one invokes. Earlier in this chapter we encountered many competing accounts of scientific understanding, so which one should we choose? Since the issue of scientific progress concerns the historical development of science, we suggest looking at the contextual theory first, since this theory is based on historical evidence and sensitive to changes in the historical context. However, this immediately leads to a problem: while the contextual theory acknowledges that understanding is, and has always been, an aim of science, it asserts that standards for the intelligibility of scientific theories - which is a necessary condition for understanding phenomena may change over the course of history, and sometimes do so in a radical way. Examples include the development of theories of gravitation from Descartes and Huygens, via Newton, to Einstein (see De Regt 2017, Chapter 5), and the Chemical Revolution, in which phlogiston theory was replaced by Lavoisier's oxygen theory of combustion (see De Regt and Gijsbers 2017). These historical developments embody radical changes in our understanding of the phenomena, which involved equally radical changes in intelligibility standards and the associated set of skills required for constructing scientific explanations. While nobody will deny that the shift from 
Newton's to Einstein's theory of gravitation constitutes scientific progress, it is less clear how this progress can be accounted for on the contextual theory of scientific understanding. It may seem that we confront a problem similar to Kuhnian incommensurability (which haunted the problem-solving account of scientific progress): How can we measure an increase of understanding if intelligibility standards change with the historical context?

An answer is provided by Finnur Dellsén (2016), who defends an account of scientific progress in terms of increasing understanding, which he defines as "grasping how to correctly explain and/or predict aspects of the target phenomenon", where grasping in turn involves the ability to anticipate the behavior of the phenomenon in a variety of circumstances (pp.74-75). Dellsén dubs his view the 'noetic' account of scientific progress and compares it with the traditional epistemic account, which explains progress in terms of an increase of knowledge (Bird 2016). He argues that the noetic account is preferable because it accommodates cases such as Einstein's explanation of Brownian motion on the basis of the kinetic theory, which feature an increase of understanding but no increase of knowledge, as well as cases in which knowledge increases but no understanding is gained. One advantage of his noetic account, Dellsén (2016, p.81) claims, is that it also explains the relevance of pragmatic virtues to scientific progress: while pragmatic virtues such as simplicity do not make a difference to the truth or propositional content of a theory, they do affect the ability to grasp explanations and predictions, and hence the understanding that can be achieved. In the contextual theory of scientific understanding the role of these pragmatic virtues is encapsulated in the definition of intelligibility, the value that promotes the epistemic aim of science: understanding.

\section{Conclusion}

In this chapter we have reviewed the main trends in the philosophical debate about scientific understanding, a debate that started relatively recently and will surely continue and expand in the years to come. Understanding scientific understanding is important for philosophers who want to understand the nature of science, but it is also of interest to a wider audience. Both (aspiring) scientists and the general public may profit from deeper insight in the way science provides us with understanding of the world around us. Today, the dominant view of science in public debates is still based on the idea that scientific research can and should uncover the truth about reality by producing knowledge of incontrovertible facts. When it turns out that real science does not achieve this, that scientists cannot deliver certain knowledge and disagree about the 'facts', the result can be a relativist or even outright anti-scientific attitude among the general public and in politics. This has happened in recent years, and according to many, we are now living in a 'post-truth society', where facts are less relevant than emotions and 'alternative facts' may shape public opinion. A more realistic view of science will help to turn the tide, and a focus on understanding as a central aim of science may play a crucial part here. Acknowledging that science does not and cannot produce certain knowledge but that its value lies rather in the understanding that it delivers, is a first step towards a philosophical account of science that has immediate societal relevance. 
Understanding why things are as they are, and understanding how the world works, is more valuable than mere knowledge of facts (if attainable at all). While the pragmatic nature of understanding perhaps detracts from its objectivity, understanding allows for prediction and control in a way that ordinary knowledge cannot, and is thereby of vital importance for the solution of societal problems. Returning to the example of climate science, mentioned in the introduction, it is clear that objective facts regarding climate change are hard to obtain: determining how exactly the climate will change on global and regional scales is an intricate matter, and even though some consensus about it may be achieved within the scientific community, as has been shown by the IPCC, it will always be possible for skeptics to throw doubt on such knowledge-claims. By contrast, scientific understanding of the process of climate change is less prone to such skeptical challenges and at the same time more useful for coping with present and future environmental problems - at least if one adopts a pragmatic conception of understanding, for example along the lines of the contextual theory. For it is the ability to use the relevant physical, chemical and biological theories to build climate models, rather than the absolute truth of these theories, that allows for prediction, manipulation, and control of the environmental system. As such, scientific understanding of the world is a prerequisite for making it a better place.

\section{References}

Baumberger, Christoph (forthcoming) Explicating objectual understanding: taking degrees seriously. Journal for General Philosophy of Science.

Baumberger, Christoph, Claus Beisbart, and Georg Brun (2017) What is understanding? An overview of recent debates in epistemology and philosophy of science. In: Grimm, Baumberger and Ammon (2017), pp.1-34.

Bird, Alexander (2016) Scientific progress. In: Paul Humphreys (ed.), The Oxford handbook of philosophy of science. New York: Oxford University Press, pp.544-563.

Cartwright, Nancy (1983) How the laws of physics lie. Oxford: Clarendon Press.

Dellsén, Finnur (2016) Scientific progress: knowledge versus understanding. Studies in History and Philosophy of Science 56, pp.72-83.

Dellsén, Finnur (2017) Understanding without justification or belief. Ratio 30, pp.239-254.

De Haro, Sebastian, and Henk W. de Regt (2018) Interpreting theories without a spacetime. European Journal for Philosophy of Science. DOI: 10.1007/s13194-018-0204-X

De Regt, Henk W. (2014) Visualization as a tool for understanding Perspectives on Science 22, pp.377-396.

De Regt, Henk W. (2015) Scientific understanding: Truth or dare? Synthese 192, pp.3781-3797.

De Regt, Henk W. (2017) Understanding scientific understanding. New York: Oxford University Press.

De Regt, Henk W., and Victor Gijsbers (2017) How false theories can yield genuine understanding. In: Grimm, Baumberger and Ammon (2017), pp.50-75. 
De Regt, Henk W., Sabina Leonelli, and Kai Eigner (eds.) (2009) Scientific understanding: philosophical perspectives. Pittsburgh: University of Pittsburgh Press.

Elgin, Catherine Z. (2017) True enough. Cambridge, MA: MIT Press.

Faye, Jan (2014) The nature of scientific thinking: on interpretation, explanation, and understanding. London: Palgrave MacMillan.

Gopnik, Alison (2000) Explanation as orgasm. Mind and Machines 8, pp.101-118.

Greco, John (2014) Episteme: Knowledge and understanding. In: Kevin Timpe and Craig A. Boyd (eds.), Virtues and their vices. Oxford: Oxford University Press, pp.285-302.

Grimm, Stephen R. (2006) Is understanding a species of knowledge? British Journal of Science 57, pp.515-535.

Grimm, Stephen R. (2009) Reliability and the sense of understanding. In: De Regt, Leonelli and Eigner (2009), pp.83-99.

Grimm, Stephen R. (2010) The goal of explanation. Studies in History and Philosophy of Science 41, pp.337-344.

Grimm, Stephen R. (2014) Understanding as knowledge of causes. In: A. Fairweather (ed.), Virtue epistemology naturalized: bridges between virtue epistemology and philosophy of science. Cham: Springer, pp.329-345.

Grimm, Stephen R. (2017) Understanding and transparency. In: Grimm, Baumberger, and Ammon 2017, pp.212-229.

Grimm, Stephen R., Christoph Baumberger, and Sabine Ammon (eds.) (2017) Explaining understanding: new perspectives from epistemology and philosophy of science. New York: Routledge.

Hills, Alison (2016) Understanding why. Noûs 50, pp.661-668.

IPCC (2013) Climate change 2013 - the physical science basis, contribution of working group I to the fifth assessment report of the IPCC. Cambridge: Cambridge University Press.

Keil, Frank (2006) Explanation and understanding. Annual Review of Psychology 57, pp.227254.

Khalifa, Kareem (2017) Understanding, explanation, and scientific knowledge. Cambridge, UK: Cambridge University Press.

Kuorikoski, Jaakko (2012) Simulation and the sense of understanding. In: C. Imbert, and P. Humphreys (eds.), Models, simulations, and representations. London: Routledge, pp.168-187.

Kvanvig, Jonathan (2003) The value of knowledge and the pursuit of understanding. New York: Cambridge University Press.

Lipton, Peter (2004) Inference to the best explanation. $2^{\text {nd }}$ ed. London: Routledge.

Lipton, Peter (2009) Understanding without explanation. In: De Regt, Leonelli and Eigner (2009), pp.43-63.

Mizrahi, Moti (2012) Idealizations and scientific understanding. Philosophical Studies 160, pp.237-252. 
Morrison, Margaret (1999) Models as autonomous agents. In: M.S. Morgan and M. Morrison (eds.), Models as mediators. Cambridge: Cambridge University Press, pp.38-65.

Newman, Mark (2012) An inferential model of scientific understanding. International Studies in the Philosophy of Science 26, pp.1-26.

Newman, Mark (2017) Theoretical understanding in science. British Journal for the Philosophy of Science 68, pp.571-595.

Potochnik, Angela (2017) Idealization and the aims of science. Chicago: The University of Chicago Press.

Pritchard, Duncan (2010) Knowledge and understanding. In: Duncan Pritchard, Alan Millar and Adrian Haddock: The nature and value of knowledge: three investigations. Oxford: Oxford University Press, pp.1-88.

Rozenblit, Leonid, and Frank Keil (2002) The misunderstood limits of folk science: an illusion of explanatory depth, Cognitive Science 26, pp.521-562.

Strevens, Michael (2013) No understanding without explanation. Studies in History and Philosophy of Science 44, pp.510-515.

Strevens, Michael (2017) How idealizations provide understanding. In: Grimm, Baumberger and Ammon (2017), pp.37-49.

Trout, J.D (2002) Scientific explanation and the sense of understanding. Philosophy of Science 69, pp.212-233.

Waskan, Jonathan, Ian Harmon, Zachary Horne, Joseph Spino, and John Clevenger (2014) Explanatory anti-psychologism overturned by lay and scientific case classifications. Synthese 191, pp.1013-1035.

Wilkenfeld, Daniel (2013) Understanding as representation manipulability. Synthese 190, pp.997-1016.

Wilkenfeld, Daniel (2017) MUDdy understanding. Synthese 194, pp.1273-1293.

Ylikoski, Petri (2009) The illusion of depth of understanding in science. In: De Regt, Leonelli and Eigner (2009), pp.100-119.

1 See De Haro and De Regt (2018) for examples from current theoretical physics.

2 See De Regt (2017, pp.31-35 and pp.103-106) for an extensive discussion of this example.

3 See De Regt (2017, pp.205-216).

4 A reason is that De Regt focusses on the 'primary' understanding that is gained when scientists discover a new explanation for a phenomenon, while most alternative accounts focus on the 'secondary' understanding that is achieved when someone comprehends an already existing explanation for a phenomenon (De Regt 2017, p.100).

5 See Kvanvig 2003; Pritchard 2010; Hills 2016; for a criticism of this view Grimm 2006; Greco 2014; Khalifa 2017, Chapter 7.

6 See Dellsén 2017; Hills 2016; Wilkenfeld 2017.

7 The hindsight bias is the effect that people systematically overestimate their predictive power in after-the-fact contexts: they remember their earlier predictions as having been more accurate than 
they actually were. The overconfidence bias is the reason why people are "systematically prone to believing that they are right when they are not" (Trout 2002, p.226).

8 One question is whether philosophers are free to adopt any notion of "proper understanding" they want, independently of empirical psychology. Waskan et al. (2014) argue against antipsychologism, on the basis of experimental evidence regarding classifications of explanations by laypeople and scientists. They suggested that philosophers of science should take seriously psychological conceptions of explanation (e.g. those that invoke considerations of intelligibility), or else run the risk of being completely out of step with everyday and scientific explanatory practice.

9 See Lipton (2004) for an analysis of inference to the best explanation; see esp. pp. 59-62 for the distinction and relation between lovely and likely explanations.

10 See Bird (2016) for a recent overview of theories of scientific progress, in which the idea that science progresses in achieving the aim of understanding is conspicuously absent. 